\title{
Iron, copper and immunocompetence
}

\author{
Carlos Muñoz ${ }^{1 *}$, Ernesto Rios ${ }^{2}$, Jorge Olivos ${ }^{3}$, Oscar Brunser $^{1}$ and Manuel Olivares ${ }^{1}$ \\ ${ }^{1}$ INTA, University of Chile, Santiago, Chile \\ ${ }^{2}$ Faculty of Medicine, Universidad Mayor, Santiago, Chile \\ ${ }^{3}$ Department of Gynaecology, Faculty of Medicine, University of Chile, Santiago, Chile
}

Microminerals including copper and iron are essential to immunity and health in human beings. The development of powerful tools in analytical cell biology and molecular genetics has facilitated efforts to identify specific cellular and molecular functions of trace elements in the maturation, activation and functions of host defence mechanisms. Selected recent reports about the role of copper and iron nutrition on immune functions are critically analysed here. Effects of trace element supplementation on infectious morbidity are also reviewed. While micromineral deficiencies, in general, may have widespread effects on nearly all components of immune response, these effects can be reversed by supplementation. However, the conflicting effects of iron deficiency and iron supplementation in vitro on the defensive systems reveals the urgent need for further additional information on the in vivo situation. In the elderly, vaccination against respiratory infections is likely to protect only $30-70 \%$ of the population. However, it may be possible to modulate immune function and ultimately reduce the severity of infections through micronutrient supplementation. Thus, microminerals contribute to the maintenance of the balance between immunity and health in humans.

Copper and Iron deficiency: Immunity: Morbidity: Supplementation

Nutrition and health are known since ancient times to be intimately linked; however, the vital influence of micronutrients on immune response was clearly established only in the last quarter of the 20th century. The understanding of adequate trace element nutrition is a key requirement to designing better nutrition that protects animals and humans against infections. The current paradigm to evaluate the status of trace element deficiencies is to characterise some effector activities in leukocytes from various tissues such as blood, bone marrow and lymphoid organs. Among others, copper and iron have so far attracted much attention, as attested by recent reviews describing the relationship between trace elements and immune functions ${ }^{1-4}$.

\section{Trace elements in the biological functions of immune cells}

The availability of powerful tools in analytical cell biology and molecular genetics has facilitated efforts to identify specific cellular and molecular functions of trace elements in the maturation, activation and functions of host defence mechanisms. Immune cells, like all other types of cell, require an adequate supply of trace elements for the structure and function of metalloproteins that participate in housekeeping processes such as energy production (e.g. iron for cytochromes $\mathrm{a}, \mathrm{b}$ and $\mathrm{c}, \mathrm{NADH}$ and succinate dehydrogenases; copper for cytochrome c oxidase in the mitochondrial electron-transport chain) and to protect the cell against highly toxic reactive oxygen species (e.g. copper for superoxide dismutase and iron for catalase). Moreover, the continuous generation of immune cells in bone marrow and the clonal expansion of lymphocytes in response to antigenic stimulation require the availability of sufficient micronutrient for the synthesis of deoxyribonucleotide precursors by ribonucleotide reductase and for the various nucleotidyl transferases that are required for DNA replication and cell division, respectively. In addition, trace elements are also required to maintain the activity of a number of enzymes that directly participate in the defence processes. The best example of such a role is the need for haem iron for myeloperoxidase-dependent generation of hypochlorous acid, which is a microbiocidal factor ${ }^{5}$. Copper and zinc, together with selenium, are linked in cytosolic defence against reactive oxygen and nitrogen species ${ }^{6}$. Undoubtedly there will be additional discoveries of metalloenzymes that are required for the normal development and reactivity of immune cells.

Trace element status can affect primary lymphoid organs including the thymus. However, the mechanism of thymus atrophy in both copper and iron deficiency is unknown. Recently, cell cycle analysis showed decreases in thymocyte proliferation, but not increases in apoptosis, as a possible cause of thymic atrophy in iron-deficient mice ${ }^{7}$. This finding differs from that observed during zinc deficiency, where apoptosis occurs in the thymus in parallel with increases in plasma cortisol levels ${ }^{8}$. It is unknown whether iron-deficient mice have elevated plasma cortisol levels. It has been established that the decrease in circulating $\mathrm{T}$ lymphocyte number is likely to be secondary to thymic atrophy. Concentrations of blood mononuclear cells from rat spleen are diminished by copper deficiency ${ }^{9}$. Iron deficiency also decreases the number of peripheral blood $\mathrm{T}$ lymphocytes where $\mathrm{T}$-helper and $\mathrm{T}$-suppressor cells are very sensitive to limited iron 
availability $^{10-12}$. Recently, our group found decreases in the numbers of naive T-helper (CD4 + CD45RA + ) and T-cytotoxic $(\mathrm{CD} 8+\mathrm{CD} 73+)$ cells in blood from iron-deficient subjects (unpublished data). This finding suggests that iron is also required for the regeneration of new $\mathrm{CD} 4+\mathrm{T}$ lymphocytes and maintenance of $\mathrm{T}$ cytolytic processes.

Micronutrients may potentially influence some processes of nonspecific immunity by modulating inflammatory cell functions. For instance, the effect of copper deficiency on phagocytic cells, particularly neutrophils and macrophages, is well detailed in the literature. Functions of neutrophils include travelling to the site of infection, adhering to the endothelium and transmigration across the endothelium, where they are involved in phagocytosis and killing of foreign invaders by activation of the respiratory burst ${ }^{13}$. Copper deficiency causes a decrease in the number of circulating neutrophils, a condition termed neutropaenia. This condition is observed in copper-deficient animals ${ }^{14}$ and humans ${ }^{15}$. Cellular copper status, respiratory burst, and candidacidal activity (yeast-killing ability) of peritoneal macrophages have been shown to decrease in severely copper deficient rats ${ }^{16}$. However, only limited understanding exists about the exact mechanisms triggered by inadequate trace element nutrition that affect macrophage function. Decreased phagocytosis by polymorphonuclear cells in iron deficient (ID) patients has recently been reported ${ }^{17}$. One possible mechanism is decreased ATPase activity in phagocyting cells resulting in altered membrane fluidity and less capability to engulf foreign particles, a process similar to that observed in rat and human ID red blood cells ${ }^{18}$

The effects of mineral deficiency on acquired immunity can be further demonstrated by examining the response of lymphocytes to $\mathrm{T}$ cell mitogens (blastogenesis). An impaired proliferative response has been reported in copper- and irondeficient animals and humans ${ }^{9,19}$. Blastogenic activity appears to be a sensitive marker of marginal trace element status and is one of the cell-mediated immune parameters that responds to mineral repletion. One of the possible mechanisms by which iron deficiency impairs lymphocyte proliferation is by reducing the translocation or activation of protein kinase $\mathrm{C}$ $(\mathrm{PKC})^{20}$. Additional evidence of a role for iron in lymphocyte signalling pathways comes from activation studies of phosphatidyl inositol-4,5-bisphosphate $\left(\mathrm{PIP}_{2}\right)$, a precursor of second messengers. Decreased $\mathrm{PIP}_{2}$ hydrolysis in activated spleen cells from Fe-deficient mice has been reported ${ }^{21}$.

Trace element status also affects the synthesis and secretion of cytokines that modulate the activities of immune and other cell types. Copper deficiency attenuates both interleukin (IL)-2 mRNA expression and protein secretion in activated Jurkat human $\mathrm{T}$ cell lines ${ }^{22}$ by inhibiting transcription of the IL-2 gene $^{23}$. However, there is a need for human trials before IL-2 production can be considered a potential marker of copper status. We recently reported that copper intake increased IL-2 production by blood cells from healthy subjects with low to normal plasma ceruloplasmin level ${ }^{24}$. Kuvibidila and co-workers ${ }^{25}$ demonstrated that iron deficiency in mice induces an imbalance in the in vivo secretion of Th1 and Th2 cytokines. Dietary iron restriction is associated with low serum levels of interferon (IFN)- $\gamma$ IL-12 and IL-10, which return to normal after iron repletion. Human studies have shown decreased levels of serum IL-2 and IL-6 in iron-deficient children ${ }^{26,27}$. These observations suggest that iron deficiency alters the balance between pro- and antiinflammatory cytokines, a change that may affect innate and cell-mediated immunity.

Human iron deficiency is a well-known public health problem that predominantly affects young children and women of childbearing age in developing countries ${ }^{28}$. The relationship between iron and immunity is complex, and has been reviewed by us and others ${ }^{1,29,30}$. Studies conducted by our group have focused the attention on the multifunctional cytokine tumor necrosis factor-alpha $\left(\mathrm{TNF}-\alpha^{31,32}\right.$, since this mediator plays important roles in both immunity and iron metabolism. We recently evaluated different TNF- $\alpha$ expression forms by activated blood mononuclear cells from adult women with ID due to chronic genital bleeding not associated with neoplasia ${ }^{33}$. Both the secretion and mRNA levels of the cytokine, but not its membrane expression, were significantly lower in ID subjects compared to the controls (Fig. 1). These data suggest that TNF- $\alpha$ secretion is transcriptionally regulated and the impaired secretion in cells from ID subjects indicate that the quality of the immune response is linked to the iron status of mononuclear cells.

To summarise the present evidence, iron deficiency depresses certain aspects of cell-mediated immunity as well as cytokine secretion/production (Table 1); humoral immunity is unaffected and the significance of hypoferraemia (as opposed to normal transferrin saturation) on the growth of microorganisms is uncertain.

\section{Trace element supplementation, infections and morbidity}

When analysing our current knowledge about the interaction between micronutrient supplementation, immunity and morbidity, several questions arise. For instance, does dietary trace element supplementation reduce infection? Or does the improvement of immunity by dietary mineral imply the recovery of health? Certainly, an evaluation of the risks and benefits of an oral mineral supplementation is of particular importance before implementing an intervention. The widespread occurrence of deficiencies of micronutrients in humans has served

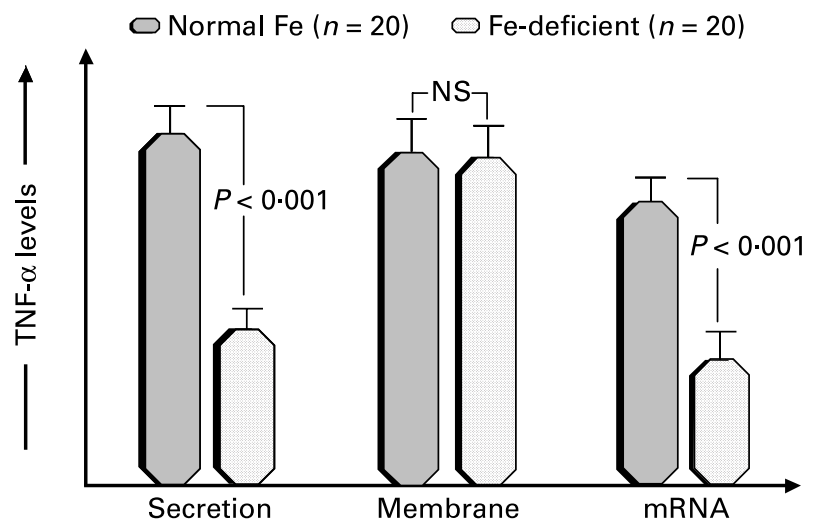

Fig. 1. TNF- $\alpha$ expression in human iron deficiency Blood mononuclear cells from iron deficient and iron replete women were stimulated and TNF- $\alpha$ protein concentration in the medium, TNF- $\alpha$ protein bound to the plasma membrane and TNF- $\alpha$ mRNA levels were measured. Data are mean \pm SEM from 20 women. Data are taken from reference 33. 
Table 1. Impaired immune functions in iron deficiency: present evidence

\begin{tabular}{llll}
\hline Species & Tissue & Immune marker examined & Reference \\
\hline Human & Plasma & IL-2 concentration & 26 (Sipahi, 1998) \\
Mouse & Spleen cells & PKC, Proliferation & 20 (Kuvibidila, 1999) \\
Mouse & Thymocytes & Proliferation & 7 (Kuvibidila, 2001) \\
Human & Blood cells & TNF- $\alpha$ transcription & 33 (Lopez, 2003) \\
Mouse & Plasma & IFN- $\gamma$ IL-12, IL-10 concentration & 25 (Kuvibidila, 2004) \\
Human & Blood cells & Phagocytic capacity & 17 (Bergmann, 2005) \\
Human & Plasma & IL-6 concentration & 27 (Ekiz, 2005) \\
Human & Blood & Lymphocyte subsets & 12 (Mullick, 2006) \\
\hline
\end{tabular}

$\mathrm{IL}=$ interleukin, $\mathrm{PKC}=$ protein kinase $\mathrm{C}, \mathrm{TNF}-\alpha=$ tumour necrosis factor, $\mathrm{IFN}-\gamma=$ interferongamma.

as the impetus to determine whether supplementation with these trace metals alone or as an adjuvant has the potential to prevent, attenuate and treat infectious diseases. However, it is also recognised that the host's needs must be balanced against the possibility that excessive amounts of redoxactive metals such as iron and copper may induce free-radical-mediated damage, and that viruses and infectious microorganisms also require the same trace elements for their survival and replication as the host. Some representative clinical trials on trace element supplementation and infectious diseases are discussed below.

Isolated human copper deficiency is difficult to find due to other concomitant macro and micronutrient deficiencies. Several years ago, our group analysed the effect of copper therapy on leukocyte phagocytosis in infantile hypocupraemia $^{15}$. Nineteen well-characterised hypocupraemic infants, 5-9 months of age, with normal weight/length ratio and free of infections, were fed daily with a cow's milk formula containing copper $(40 \mu \mathrm{g} / \mathrm{kg})$ for one month. Plasma copper and ceruloplasmin concentrations, and phagocytic activity of polymorphonuclear leukocytes were measured before and after therapy. After supplementation, copper and ceruloplasmin concentrations as well as the phagocytic index recovered to normal values. However, further clinical trials analysing the effect of copper supplementation in subjects with different infections are needed.

Since iron deficiency anaemia is a major worldwide health problem especially in malaria infected children from developing countries, several investigators have analysed the effect of supplementation of this micronutrient on infectious morbidity in randomised, double blind placebo-controlled trials ${ }^{34-38}$. Most of these studies recorded important laboratory outcomes of the intervention, such as haemoglobin change and parasite prevalence. For instance, Berger and co-workers ${ }^{37}$ assessed the impact of a 3-month daily oral Fe supplementation on haematological status, cell-mediated immunity and susceptibility to infections in 6-36 month-old children from rural Africa living in an environment where $\mathrm{Fe}$ deficiency and malaria are frequent. Iron supplementation had significant and positive effects on iron status and some immune factors since haemoglobin and total $\mathrm{T}$ and $\mathrm{Th}$ cells were improved post-therapy. However, no impact on the incidence of malaria was found. These data suggest that control of ID by oral Fe supplementation in young children has to be conducted in association with prophylaxis and treatment of malaria and repeated deworming. Special series of papers dealing with micronutrient supplements on anaemia, growth and morbidity during infancy have been published ${ }^{39-42}$. These studies were carried out by the International Research on Infant Supplementation in 4 countries (Vietnam, Indonesia, Peru and South Africa) using a common protocol and data collection instruments. The daily administration of a multiple micronutrient supplement is more efficacious in improving micronutrient status, anaemia, and child weight growth than daily iron supplement or weekly multiple micronutrient supplements. However, the collected morbidity data show no difference between any supplement group and the placebo group. More recently, iron plus zinc supplementation, but not iron alone, was found to be protective against $P$. vivax in children younger than five years old in the Peruvian Amazon ${ }^{43}$. Further research should also investigate whether there are combined benefits from the use of multiple micronutrient therapy in parallel with maternal and infant supplementations together with infection control measures. Additional controlled clinical studies are clearly warranted before definitive recommendations are made. Studies are also urgently needed to assess the effect of ID and iron supplementation on morbidity and mortality due to HIV, tuberculosis and typhoid fever.

Another interesting application of micronutrient intervention is related to vaccine response in older people, since mortality associated with influenza is more likely to occur in this population. The report of UK National Diet and Nutritional Survey found that up to $40 \%$ of older, institutionalised people had low biochemical indices for certain micronutrients. The effect of an experimental nutritional formula on symptoms of upper respiratory tract infection (URTI) and immune function in healthy seniors has been investigated ${ }^{44}$. It was found that subjects consuming the formula had significantly fewer days of symptoms of URTI, better antibody response to influenza, and greater lymphocyte proliferative response to influenza vaccine components postimmunization than control subjects. This study concludes that in an elderly population, in which only $30-70 \%$ of those vaccinated are likely to be protected from hospitalization due to influenza, it may be possible to modulate immune function and ultimately reduce symptoms through micronutrient supplementation. Since nutritional intervention may be important in improving seroconversion, Allsup and associates ${ }^{45}$ recently investigated whether a short period of micronutrient therapy could improve antibody response to the influenza vaccine when administered to older people living in long-term facilities. One hundred and sixty-four residents aged 60 and older were randomised to receive either micronutrient supplement or placebo (one tablet twice a day for 8 weeks); the influenza 
vaccine was administered 4 weeks after their commencement. Results of seroconversion to each of the 3 antigens in the 2000/2001 influenza vaccine (serotypes: H1N1, H3N2, B) showed no differences between the supplemented and placebo groups. Thus, short micronutrient supplementation had no beneficial effect on the antibody response to the influenza vaccine. Larger trials are needed to investigate the effects of long-term therapy and clinical outcome in poorly nourished institutionalised older people.

\section{Possible future directions}

Micronutrient deficiencies are of clinical and public health importance in developing countries. Studies to date on micronutrients indicate they have as much potential to alter innate immunity as macronutrients do. In supplementation studies, micronutrients may have varied effects on inflammatory cells that may be either direct or indirect, making it difficult to link in vitro observations with those studies. While micronutrient deficiency, in general, may have a widespread effect on nearly all components of the innate immune response, that effect can be reversed by supplementation. Although preliminary work is promising, an extensive number of well-controlled studies need to be done to clarify which micronutrients (alone or in combination), and in what concentrations, are necessary to influence immunity. It would also be important to select appropriate methods and parameters for accurate assessment. Thus, an important area of focus should be on innate immunity, micronutrient status, and disease.

On the other hand, deficiencies of trace elements affect almost one-third of all elderly subjects. It is expensive and impractical to estimate dietary intake or blood levels of various nutrients in such individuals. Since there is no evidence to suggest that physiological amounts of trace elements given for prolonged periods have any toxic or adverse consequences, and given the high prevalence of deficiencies of several micronutrients in old age, it would be prudent to opt for a suitable micronutrient supplement in modest amounts for all elderly individuals in order to achieve the maximum physiological and health benefits with the least risk of toxicity.

Thus, a better understanding of the molecular and cellular changes caused by inadequate trace elements should lead to the development of effective immunotherapeutic interventions and to an improvement in quality of life for many human beings.

\section{Conflict of interest statement}

This study was supported by Fondecyt grant 1020693 and ENL 06/9. The authors have no conflicts of interest to report. All authors participated in the writing of the manuscript.

\section{References}

1. Brock JH \& Mulero V (2000) Cellular and molecular aspects of iron and immune function. Proc Nutr Soc 59, 537-540.

2. Erickson KL, Medina EA \& Hubbard NE (2000) Micronutrients and innate immunity. J Infec Dis 182, Suppl 1, S5-S10.

3. Bonham M, O'Connor JM, Hannigan BM \& Strain JJ (2002) The immune system as a physiological indicator for marginal copper status? Br J Nutr 87, 393-403.
4. Cunningham-Rundles S, Ahrn S, Abuav-Nussbaum R \& Dnistrian A (2002) Development of immunocompetence: role of micronutrients and microorganisms. Nutr Rev 60, S68-S72.

5. Hampton MB, Kettle AJ \& Winterbourn CC (1998) Inside the neutrophil phagosome: oxidants, myeloperoxidase, and bacterial killing. Blood 92, 3007-3017.

6. Klotz LO, Kröncke KD, Buchczyk DP \& Sies H (2003) Role of copper, zinc, selenium and tellurium in the cellular defense against oxidative and nitrosative stress. $J$ Nutr 133, 1448S-1451S.

7. Kuvibidila SR, Porretta C, Baliga BS \& Leiva LE (2001) Reduced thymocyte proliferation but not increased apoptosis as a possible cause of thymus atrophy in iron-deficient mice. Br J Nutr 86, 157-162.

8. Fraker PJ, King LE, Laakko T \& Vollmer TL (2000) The dynamic link between the integrity of the immune system and zinc status. $J$ Nutr 130, 1399S-1406S.

9. Bala S, Failla ML \& Lunney JK (1991) Alterations in splenic lymphoid cell subsets and activation antigens in copperdeficient rats. J Nutr 121, 745-753.

10. Santos PC \& Falcao RP (1990) Decreased lymphocyte subsets and NK cell activity in iron deficiency anemia. Acta Hematol 84, 118-121.

11. Kemp JD (1993) The role of iron and iron binding proteins in lymphocyte physiology and pathology. J Clin Immunol 13, 81-92.

12. Mullick S, Rusia U, Sikka M \& Faridi MA (2006) Impact of iron deficiency anaemia on $\mathrm{T}$ lymphocytes \& their subsets in children. Indian J Med Res 124, 647-654.

13. Karimbakas J, Langkamp-Henken B \& Percival SS (1998) Arrested maturation of granulocytes in copper deficient mice. J Nutr 128, 1855-1860.

14. Koller LD, Mulhern SA, Frankel NC, Steven MG \& Williams JR (1987) Immune dysfunction in rats fed a diet deficient in copper. Am J Clin Nutr 45, 997-1006.

15. Heresi G, Castillo-Duran C, Munoz C, Arevalo M \& Schlesinger L (1985) Phagocytosis and immunoglobulins levels in hypocupremic infants. Nutr Res 5, 1327-1334.

16. Babu U \& Failla ML (1990) Respiratory burst and candidacidal activity of peritoneal macrophages are impaired in copperdeficient rats. J Nutr 120, 1692-1699.

17. Bergmann M, Hertzel S, Pinchasi R, Straussberg R, Djaldetti M \& Bessler H (2005) Phagocytic capacity and apoptosis of peripheral blood cells from patients with iron deficiency anemia. Biomed Phamacother 59, 307-311.

18. Ifere GO, Ifon ET, Ebong PE \& Umoh IB (1996) Abnormalities in adenosine tryphosphatase of the erythrocyte membrane in iron deficiency. J Trace Elem Med Biol 10, 185-188.

19. Kelley DS, Daudu PA, Taylor PC, Mackey BE \& Turnlund JR (1995) Effects of low copper diets on human immune response. Am J Clin Nutr 62, 412-416.

20. Kuvibidila SR, Kitchens D \& Baliga BS (1999) In vivo and in vitro deficiency reduces protein kinase $\mathrm{C}$ activity and translocation in murine splenic and purified T cells. J Cell Biochem 74, $468-478$.

21. Kuvibidila SR, Baliga SB, Warrier RP \& Suskind RM (1998) Iron deficiency reduces the hydrolysis of cell membrane phosphatidyl inositol-4,5-bisphosphate during splenic lymphocyte activation in C57BL/6 mice. J Nutr 128, 1077-1083.

22. Hopkins RG \& Failla ML (1997) Copper deficiency reduces interleukin-2 (IL-2) production and IL-2 mRNA in human T-lymphocytes. J Nutr 127, 257-262.

23. Hopkins RG \& Failla ML (1999) Transcriptional regulation of interleukin-2 gene expression is impaired by copper deficiency in Jurkat human T lymphocytes. J Nutr 129, 596-601.

24. Munoz C, Lopez M, Olivares M, Pizarro F, Arredondo M \& Araya M (2005) Differential response of interleukin-2 
production to chronic copper supplementation in healthy humans. Eur Cyt Network 16, 261-265.

25. Kuvibidila SR \& Warrier RP (2004) Differential effects of iron deficiency and underfeeding on serum levels of interleukin-10, interleukin-12p40, and interferon-gamma in mice. Cytokine 26, 73-81.

26. Sipahi T, Akar N, Egin Y \& Cin S (1998) Serum interleukin-2 and interleukin-6 levels in iron deficiency anemia. Pediatr Hematol Oncol 15, 69-73.

27. Ekiz C, Agaoglu L, Karakas Z, Gurel N \& Yalcin I (2005) The effect of iron deficiency anemia on function of the immune system. Hematol J 5, 579-583.

28. Ramakrishnan U (2002) Prevalence of micronutrient malnutrition worldwide. Nutr Rev 60, S46-S52.

29. Walter T, Olivares M, Pizarro F \& Munoz CIron, anemia, infection (1997) Nutr Rev 55, 111-124.

30. Oppenheimer SJ (2001) Iron and its relation to immunity and infectious disease. J Nutr 131, 616S-636S.

31. Munoz C, Rios E, Lopez M, Schlesinger L \& Nunez MT (1999) Transferrin and iron salts modulate differently tumor necrosis factor- $\alpha$ secretion by cultured human mononuclear cells. Nutr Res 19, 651-661.

32. Munoz C \& Olivares M (2000) Transferrin modulates TNF- $\alpha$ secretion by cultured human mononuclear cells: influence of iron status. Nutrition 16, 229-230.

33. Lopez M, Rios E, Schlesinger L, Olivares M, Nunez MT \& Munoz C (2003) Tumour necrosis factor-(transcription in transferrin-stimulated human blood mononuclear cells: is transferrin receptor involved in the signalling mechanism? $\mathrm{Br} J$ Haematol 120, 829-835.

34. Harvey P, Heywood P, Nesheim MC, et al. (1989) The effect of iron therapy on malarial infection in Papua New Guinean school children. Am J Trop Med Hyg 40, 12-18.

35. Smith AW, Hendrickse RG, Harrison C, Hayes RJ \& Greenwood BM (1989) The effects on malaria of treatment of iron-deficiency anaemia with oral iron in Gambian children. Trans Trop Paediatr 9, 17-23.

36. van den Hombergh J, Dalderop E \& Smith Y (1996) Does iron therapy benefit children with severe malaria-associated anaemia? A clinical trial with 12 weeks supplementation of oral iron in young children from the Turlani Division, Tanzania. J Trop Pediatr 42, 220-227.

37. Berger J, Dyck JL, Aplogan A, Schneider D, Traissac P \& Hereberg S (2000) Effect of daily iron supplementation on iron status, cell-mediated immunity, and incidence of infections in 6-36 month old Togolese children. Eur J Clin Nutr 54, 29-35.

38. Shankar AH (2000) Nutritional modulation of malaria morbidity and mortality. $J$ Infect Dis 182, S37-S53.

39. Untoro J, Karyadi E, Wibowo L, Erhardt MW \& Gross R (2005) Multiple micronutrient supplements improve micronutrient status and anemia but not growth and morbidity of Indonesian infants: a randomized, double-blind, placebo-controlled trial. J Nutr 135, 639S-645S.

40. Lopez G, Cusirramos S, Lopez D \& Gross R (2005) Efficacy of multiple supplementation for improving anemia, micronutrient status, growth and morbidity of Peruvian infants. J Nutr 135, 646S-652S.

41. Smuts CM, Dhansay MA, Faber M, et al. (2005) Efficacy of multiple micronutrient supplementation for improving anemia, micronutrient status, and growth in South African infants. $J$ Nutr 135, 653S-659S.

42. Hop LT \& Berger J (2005) Multiple micronutrient supplementation improves anemia, micronutrient status, and growth of Vietnamese infants: double-blind, randomized, placebo-controlled trials. $J$ Nutr 135, 660S-665S.

43. Richard SA, Zavaleta N, Caulfield LE, Black RE, Witzing RS \& Shankar AH (2006) Zinc and iron supplementation and malaria, diarrhea, and respiratory infections in children in the Peruvian Amazon. Am J Trop Med Hyg 75, 126-132.

44. Langkamp-Henken B, Bender BS, Gardner EM, HerrlingerGarcia KA, Kelley MJ, Murasko DM, et al. (2004) Nutritional formula enhanced immune function and reduced days of symptoms of upper respiratory tract infection in seniors. J Am Geriatr Soc 52, 3-12.

45. Allsup SJ, Shenkin A, Gosney MA, Taylor S, Taylor W, Hammond M \& Zambon MC (2004) Can a short period of micronutrient supplementation in older institutionalized people improve response to influenza vaccine? A randomized, controlled trial. J Am Geriatr Soc 52, 20-24. 